\title{
DÜBLIN
}

Technological University Dublin

ARROW@TU Dublin

2012-05-04

\section{Diacetone acrylamide-based non-toxic holographic photopolymer}

\author{
Dervil Cody \\ Technological University Dublin, dervil.cody@tudublin.ie \\ Izabela Naydenova \\ Technological University Dublin, izabela.naydenova@tudublin.ie \\ Emilia Mihaylova \\ Technological University Dublin, emilia.mihaylova@tudublin.ie
}

Follow this and additional works at: https://arrow.tudublin.ie/cieocon2

Part of the Optics Commons

\section{Recommended Citation}

D. Cody, I. Naydenova \& E. Mihaylova (2012) Diacetone acrylamide-based non-toxic holographic photopolymer, Proc. SPIE 8429, 84291B; http://dx.doi.org/10.1117/12.923116

This Conference Paper is brought to you for free and open access by the Centre for Industrial and Engineering Optics at ARROW@TU Dublin. It has been accepted for inclusion in Conference Papers by an authorized administrator of ARROW@TU Dublin. For more information, please contact arrow.admin@tudublin.ie, aisling.coyne@tudublin.ie, gerard.connolly@tudublin.ie. Funder: Irish Research Council for Science, Engineering and Technology 


\title{
Diacetone acrylamide-based non-toxic holographic photopolymer
}

\author{
D. Cody, ${ }^{1,2}$ I. Naydenova, ${ }^{1,2}$ E. Mihaylova ${ }^{1,2,3, *}$ \\ ${ }^{1}$ Centre for Industrial and Engineering Optics, Dublin Institute of Technology, Dublin 8, Ireland \\ ${ }^{2}$ School of Physics, College of Science and Health, Dublin Institute of Technology, Dublin 8, Ireland \\ ${ }^{3}$ Department of Mathematics and Physics, Agricultural University, Plovdiv, Bulgaria
}

\begin{abstract}
A new low-toxicity diacetone acrylamide-based photopolymer is developed and characterized. The environmentallycompatible photopolymer has been modified with the inclusion of glycerol. The incorporation of glycerol results in a uniform maximum refractive index modulation for recording intensities in the range of $1-20 \mathrm{~mW} / \mathrm{cm}^{2}$. This may be attributed to glycerol's nature as a plasticizer, which allows for faster diffusion of un-reacted monomer within the grating during holographic recording. An optimum recording intensity of $0.5 \mathrm{~mW} / \mathrm{cm}^{2}$ is observed for exposure energies of 20 $60 \mathrm{~mW} / \mathrm{cm}^{2}$. The modified photopolymer achieves a refractive index modulation of $2.2 \times 10^{-3}$, with diffraction efficiencies up to $90 \%$ in $100 \mu \mathrm{m}$ layers. The photopolymer layers containing glycerol have improved stability and optical quality.
\end{abstract}

Keywords: non-toxic photopolymer, holography, diffraction gratings

\section{INTRODUCTION}

The optimization and characterization of a new non-toxic Diacetone Acrylamide (DA) based photopolymer has previously been described ${ }^{1}$. The improved photopolymer composition is shown to have holographic recording features similar to that of the standard Acrylamide (AA) based photopolymer ${ }^{2}$, but with reduced toxicity due to the replacement low-toxicity monomer Diacetone Acrylamide ${ }^{3-9}$. It has also shown to surpass the holographic recording capabilities of other recently developed low toxicity photopolymer materials ${ }^{10-12}$. This materials non-toxic nature, wide dynamic range and high holographic sensitivity, together with its self-processing nature and low cost of production, make it an excellent candidate for applications such as holographic sensors, diffractive optics and in data storage.

This paper details the effect of the addition of glycerol on the holographic recording properties of the new DA photopolymer. Glycerol is a transparent, viscous liquid which is reported to have low-toxicity ${ }^{13}$. It is widely used in the food and pharmaceutical industries as a solvent and lubricant. Glycerol has been shown to influence the maximum refractive index modulation's dependence on recording intensity, the low-intensity response of the material, as well as the stability of the DA photopolymer samples.

\section{THEORY}

A typical photopolymer which reacts via a radical chain mechanism consists of a main monomer, a cross-linking monomer, an electron donor, a photosensitising dye, and a binder matrix, which holds all of the components together. These photopolymer components are spatially redistributed during holographic recording due to the polymerization reaction which occurs in the material on exposure to light. This reaction involves three steps: initiation, propagation and termination. When illuminated, dye molecules absorb photons of light, and are promoted to excited singlet states.

$$
X D+h v \rightarrow^{1} X D \bullet
$$

These singlet states can re-emit this energy via fluorescence, or by radiationless energy transfer to another molecule. 
${ }^{1} X D \bullet \rightarrow X D+h v$

${ }^{1} X D \bullet+Y \rightarrow X D+Y$

Alternatively, the singlet state can be converted to the more stable and longer lived excited triplet state dye molecule, via intersystem crossing.

${ }^{1} X D \bullet \rightarrow{ }^{3} X D \bullet$

This triplet state dye molecule then reacts with the electron donor to produce a pair of radicals.

${ }^{3} X D \bullet+E D \rightarrow X D+E D \bullet$

These radicals react with the monomer to produce an initiating species.

$E D \bullet+M \rightarrow E D-M \bullet$

The growing chain continues to add more monomer units via propagation.

$E D-M \bullet+M \rightarrow E D(M)_{2}$

This propagation step will continue until one of two termination reactions occurs, namely combination or disproportionation.

Oxygen quenching is another important process. It causes a reduction in the yield of singlet and triplet state dye molecules.

$$
\begin{aligned}
& { }^{3} \mathrm{XD} \bullet+{ }^{3} \mathrm{O}_{2} \rightarrow \mathrm{XD}+{ }^{3} \mathrm{O}_{2} \bullet \\
& { }^{1} \mathrm{XD} \bullet+{ }^{3} \mathrm{O}_{2} \rightarrow \mathrm{XD}+{ }^{1} \mathrm{O}_{2} \bullet
\end{aligned}
$$

Oxygen-quenching can cause an inhibition period at the start of polymerization, and can lead to a reduction in the rate of photo-bleaching of the layer. Most of the oxygen in the layer must be used up during holographic exposure before polymerization can begin ${ }^{14}$. Glycerol is a known reducing agent and has been shown to stimulate the level of oxygenation in certain materials ${ }^{15-16}$. The effect of glycerol on photosensitive systems has been studied previously. Meyer et al. ${ }^{17}$ and Galassi ${ }^{18}$ report that increasing the concentration of glycerol causes up to an order of magnitude drop in the photobleaching rate for two very different photosensitive systems. Slower rates of photobleaching are desirable for holographic photopolymers, as less dye molecules are bleached initially, allowing for longer exposure times before saturation is reached. This allows for the formation of longer polymer chains and hence a higher refractive index modulation. Glycerol is reported to have a pronounced effect the sensitivity of photopolymer systems also ${ }^{16}$.

After preliminary optimization, the optimum concentration of glycerol of in the stock photopolymer solution ${ }^{1}$ was found to be $3.85 \%$ vol/vol. The modified photopolymer (DAG) has been characterized in terms of its refractive index modulation dependence on recording intensity, exposure energy and spatial frequency of recording. The modified composition has been compared with the original DA composition (DA0). 


\section{EXPERIMENT:}

\subsection{Preparation of photopolymer layers}

The two photopolymer compositions tested were prepared as described in Table 1 . The photopolymer solution was then deposited on to glass slides $(76 \times 26 \mathrm{~mm})$ and allowed to dry for 12-24 hours in darkness under normal laboratory conditions $\left(20-25^{\circ} \mathrm{C}, 40-60 \% \mathrm{RH}\right)$. Layer thickness was measured using a white-light surface profiler (Micro XAM S/N 8038).

\subsection{Experimental set-up}

A two-beam holographic optical setup (see Figure 1) with an angle of $30^{\circ}$ between the beams was used to record unslanted transmission gratings using a 532nm Nd:YVO 4 laser. Gratings were recorded in the layers with exposure energies of $20-100 \mathrm{~mJ} / \mathrm{cm}^{2}$ at a spatial frequency of $1000 \pm 10$ lines $/ \mathrm{mm}$. The absorption of the photopolymer at $633 \mathrm{~nm}$ is negligible, so a $633 \mathrm{~nm}$ He-Ne laser was used as the probe beam at the Bragg angle. An optical power meter (Newport 1830-C) was used to record the intensity of the diffracted beam and LabVIEW software was used to plot the data in realtime. In order to measure the diffracted intensity dependence on the incident angle of the probe beam, the grating was placed on a rotational stage (Newport, ESP 300).

Table 1. Composition of DA photopolymer solutions.

\begin{tabular}{|l|l|l|}
\hline \multicolumn{3}{|c|}{ Photopolymer Compositions } \\
\hline & DA0 & DAG \\
PVA 10\% wt/vol (ml) & 20 & 20 \\
TEA (ml) & 2 & 2 \\
Diacetone Acrylamide (g) & 1 & 1 \\
Bisacrylamide (g) & 0.2 & 0.2 \\
Erythrosin B 0.11\% wt/vol & 4 & 4 \\
(ml) & & \\
Glycerol (ml) & - & 1 \\
\hline
\end{tabular}

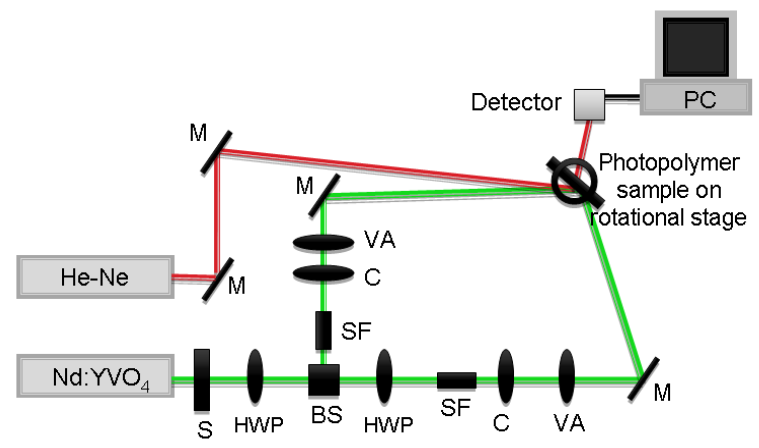

Figure 1. Experimental setup: S: shutter, HWP: half wave plate, BS: polarizing beam splitter, SF: spatial filter, C: collimator, VA: variable aperture, M: mirror. 


\section{RESULTS AND DISCUSSION:}

\subsection{Dependence of refractive index modulation on recording intensity}

The intensity dependence of the modified photopolymer at $1000 \mathrm{l} / \mathrm{mm}$ was investigated. Transmission gratings were recorded in photopolymer layers using a recording intensity which was varied from $1-5 \mathrm{~mW} / \mathrm{cm}^{2}$, with constant exposure energy of $100 \mathrm{~mJ} / \mathrm{cm}^{2}$. As can be seen from figure 2, while DA0 (composition containing no glycerol) has an obvious optimum intensity at $2 \mathrm{~mW} / \mathrm{cm}^{2}$, the intensity dependence for DAG (composition containing glycerol) is approximately uniform for the range of intensities tested. This is probably due to glycerol's nature as a plasticizer, which allows the unreacted monomer to diffuse more quickly into the illuminated regions within the layer. For the composition DA0 which contains no glycerol, there is more obvious intensity dependence as the monomer moves slower. The decrease in refractive index modulation for the DAG composition containing glycerol can be explained by the lower percent weight of monomer in the modified composition. Refractive index modulation matching that of the DA composition without glycerol has been obtained by increasing the percent weight of overall monomer from $17.31 \%$ to $23.25 \%$. However this results in a reduction in the optical quality of the photopolymer layers, and therefore repeatability of results is an issue. The composition will need to be further optimized to improve the optical quality for higher monomer concentrations.

\subsection{Dependence of refractive index modulation on exposure energy}

As is shown in figure 3, DAG reaches an optimum refractive index modulation at $0.5 \mathrm{~mW} / \mathrm{cm}^{2}$, for the lower exposure energies $\left(20-60 \mathrm{~mJ} / \mathrm{cm}^{2}\right)$. This is not as prominent at the higher exposure energies of $80-100 \mathrm{~mJ} / \mathrm{cm}^{2}$. This can be explained by considering the key factor that contributes to grating formation; the ratio of the rate of polymerization to the rate of monomer diffusion ${ }^{19}$.

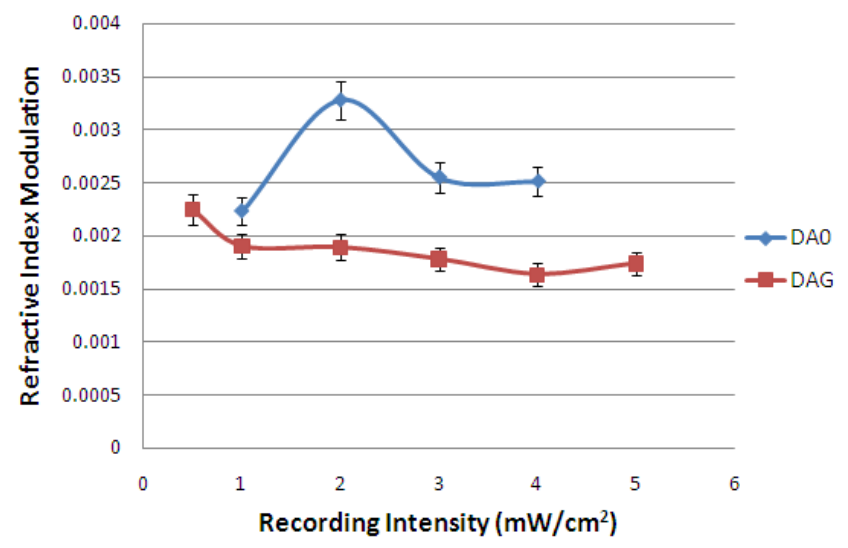

Figure 2. Refractive index modulation vs. recording intensity for the DA0 (no glycerol), DAG (with glycerol) compositions at $1000 \mathrm{l} / \mathrm{mm}$ for an exposure energy of $100 \mathrm{~mJ} / \mathrm{cm}^{2}$. 


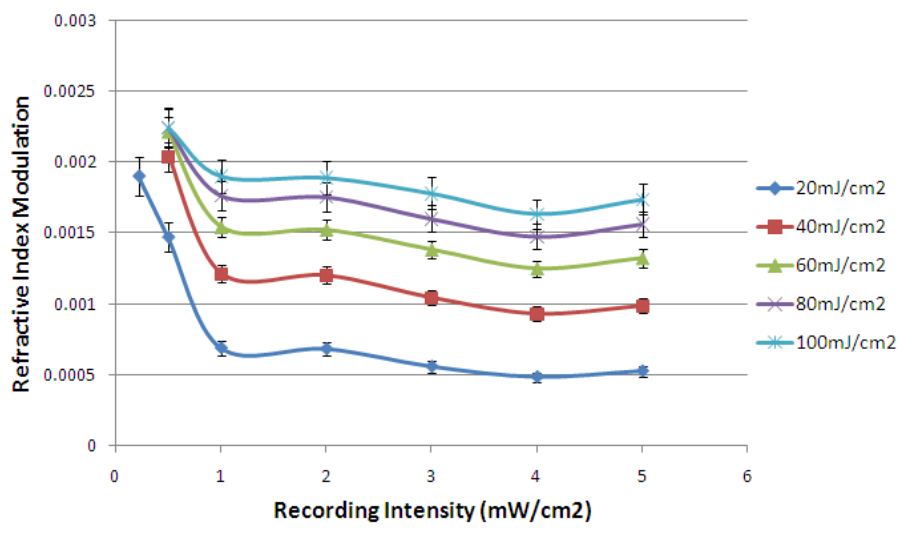

Figure 3. Refractive index modulation vs. recording intensity for DAG over a range of exposure energies.

At lower recording intensities, the rate of polymerization is slow due to the low number of initiating species. Therefore the increased rate of diffusion due to the addition of glycerol ensures that the maximum refractive index modulation is reached quickly, as the un-reacted monomer molecules can easily diffuse into the illuminated areas and be polymerized. This explains the trend seen in figure 3. There is a more obvious optimum recording intensity of $0.5 \mathrm{~mW} / \mathrm{cm}^{2}$ at the lower exposure energies $\left(20-60 \mathrm{~mJ} / \mathrm{cm}^{2}\right)$, as the exposure times needed to reach the maximum refractive index modulation are shorter. This is possibly due to the increased rate of diffusion relative to the rate of polymerization, caused by glycerol. Therefore the refractive index modulation is higher at the lower exposure energies of $20-60 \mathrm{~mJ} / \mathrm{cm}^{2}$ for a recording intensity of $0.5 \mathrm{~mW} / \mathrm{cm}^{2}$. As the intensity is increased beyond $0.5 \mathrm{~mW} / \mathrm{cm}^{2}$, the rate of polymerization overtakes the rate of diffusion, and so the maximum refractive index modulation achieved decreases and levels out.

Higher recording intensities of 10 and $20 \mathrm{~mW} / \mathrm{cm}^{2}$ were then investigated, and the results of this are shown in figure 4. The value for refractive index modulation decreases by a maximum of $8.93 \%$ as the recording intensity is increased from $2-20 \mathrm{~mW} / \mathrm{cm}^{2}$ over the range of exposure energies tested. The uniform intensity response of the DA material is a desirable feature for holographic applications. The use of high recording intensities allows for much faster recording, which is necessary for hologram production on a large scale.

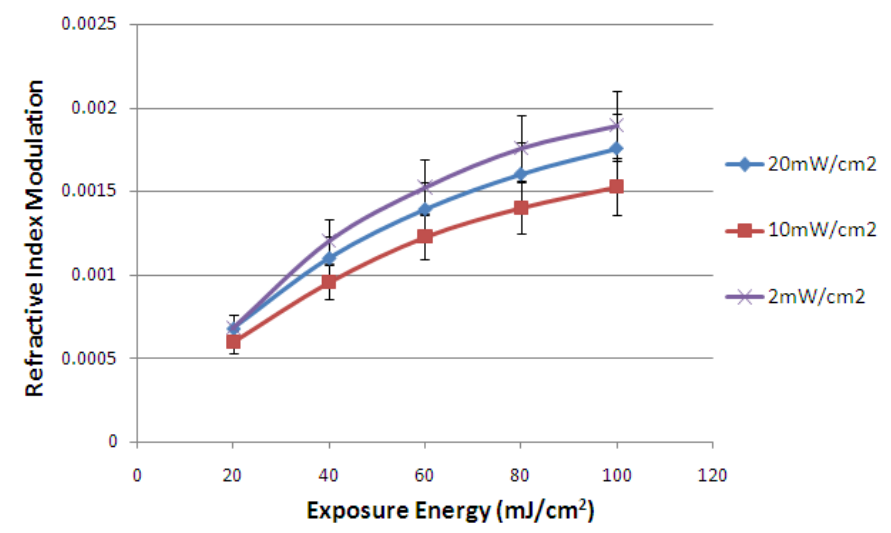

Figure 4. Refractive index modulation vs. exposure energy for DAG samples tested at low $\left(2 \mathrm{~mW} / \mathrm{cm}^{2}\right)$ and high $(10$ and 20 $\mathrm{mW} / \mathrm{cm}^{2}$ ) intensities. 


\subsection{Dependence of refractive index modulation on spatial frequency}

The maximum refractive index modulation for different recording intensities was investigated for the DAG photopolymer at $3000 \mathrm{l} / \mathrm{mm}$. The result of this is shown in figure 5 . At the higher spatial frequency of $30001 / \mathrm{mm}$, the maximum refractive index modulation achievable for DAG falls off, as is also the case for DA0. DAG reaches a maximum RIM of $\sim 3.5 \times 10^{-4}$, which is lower than that achieved with the DA0 composition. However DAG retains its linear intensity dependence over the range of recording intensities tested.

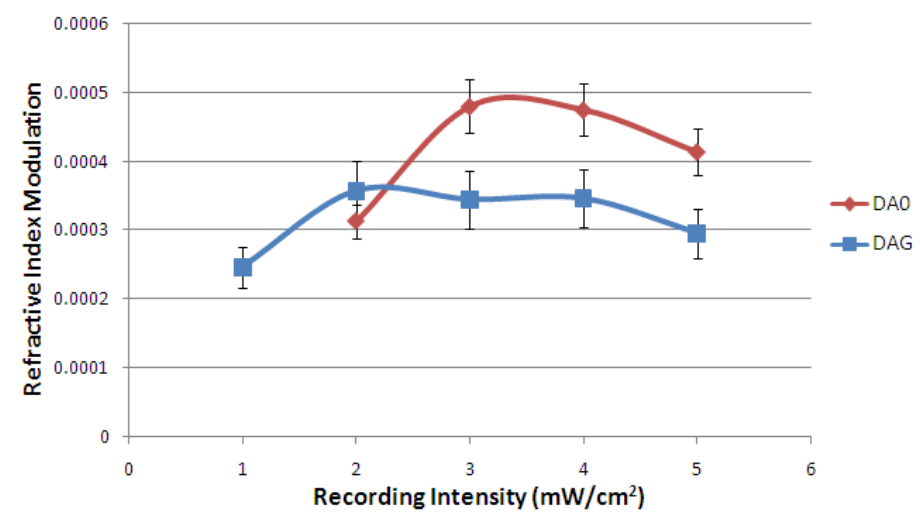

Figure 5. Refractive index modulation vs. recording intensity for the DA0 (no glycerol) and DAG (with glycerol) compositions at $3000 \mathrm{1} / \mathrm{mm}$ for an exposure energy of $100 \mathrm{~mJ} / \mathrm{cm}^{2}$.

\section{CONCLUSION:}

The effect of the addition of glycerol to a new non-toxic holographic photopolymer material has been studied. Addition of glycerol to the photopolymer composition causes the maximum refractive index modulation at low recording intensities to be reached quickly. This implies that the diffusion rates are increased. Glycerol has also shown to improve the stability and optical quality of the photopolymer layers. Further optimization of the percent weight of monomer will be carried out, in order to optimize the maximum achievable refractive index modulation of the modified composition. Characterization of the new material's holographic recording capabilities in the reflection mode is currently being carried out.

The authors acknowledge the Irish Research Council for Science, Engineering and Technology (IRCSET) for financial support, and also thank the FOCAS Research Institute for providing the research facilities to carry out the experimental work.

*corresponding author e-mail: emilia.mihaylova@dit.ie

\section{REFERENCES}

[1] Cody, D., Naydenova, I. and Mihaylova, E., "New non-toxic holographic photopolymer," J.Opt. 14(1), 015601 (2012).

[2] Martin, S., Leclere, P., Renotte, Y., Toal V. and Lion, Y., "Characterisation of an acrylamide-based dry photopolymer holographic recording material," Opt. Eng. 33(12), 3942-3946 (1995).

[3] McCollister, D. D., Oyen, F. and Rowe, V. K., “Toxicology of Acrylamide," Toxicol. Appl. Pharm. 6(2), 172-181 (1964).

[4] Lawrence, A. G., Gentry, R., McDonald, T., Bartow, H., Bounds, J., Macdonald, N., Clewell, H., Allen B. and Van Landingham, C., "Acrylamide: Review of Toxicity Data and Dose-Response Analyses for Cancer and Noncancer Effects," Crit.Rev. Toxicol. 36(6-7), 481-608 (2006). 
[5] King, D. J. and Noss R. R., “Toxicity of polyacrylamide and acrylamide monomer,” Rev. Environ. Health 8(1-4), 316 (1989).

[6] Health implications of acrylamide in food: report of a joint FAO/WHO consultation, WHO Headquarters, Geneva, Switzerland, 25-27 June, 2002.

[7] Siemiatycki, J., Richardson, L., Straif, K., Latreille, B., Lakhani, R., Campbell, S., Rousseau M. and Boffetta, P., "Listing Occupational Carcinogens," Environ. Health Perspect. 112, 1447-1459 (2004).

[8] Acrylamide; Sigma-Aldrich; http://www.sigmaaldrich.com/catalog/DisplayMSDSContent.do (accessed 30/6/11).

[9] Diacetone Acrylamide; Sigma-Aldrich; http://www.sigmaaldrich.com/catalog/DisplayMSDSContent.do (accessed 30/6/11).

[10] Ortuno, M., Fernandez, E., Gallego, S., Belendez, A. and Pascual, I.,"New photopolymer holographic recording material with sustainable design," Opt. Express 15(19), 12426-12435 (2007).

[11] Gallego, S., Marquez, A., Ortuno, M., Marini, S. and Frances, J., "High environmental compatibility photopolymers compared to PVA/AA based materials at zero spatial frequency limit," Opt. Mater. 33(3), 531-537 (2011).

[12] Olivares-Perez, A., Hernandez-Garnay, M. P., Fuentes-Tapia, I. and Ibarra-Torres, J. C., "Holograms in polyvinyl alcohol photosensitized with $\mathrm{CuCl}_{2}\left(\mathrm{H}_{2} \mathrm{O}\right)$," Opt. Eng. 50(6), 0658011-0658016 (2011).

[13] Sanderson, D. M., “A note on glycerol formal as a solvent in toxicity testing,” J. Pharm. Pharmacol. 11(1), 150-156 (1959).

[14] Pavani, K., "Holographic Liquid Crystal Devices," Thesis (PhD), Dublin Institute of Technology (2009).

[15] Ren, X., Yang, Z. and Kuang, T., "Solvent-induced changes in photochemical activity and conformation of photosystem 1 particles by glycerol," Biol. Chem. 387(1), 23-29 (2006).

[16] Bennett, C. W., "Glycerol as Sensitizer,” J. Phys. Chem. 16(7), 614-615 (1911).

[17] Meyer, T., Tollin, G., Hazzard, J. and Cusanovich, M.,"Photoactive yellow protein from the purple phototropic bacterium," Biophys. J. 56(3), 559-564 (1989).

[18] Galassi, L., "Wavelength dependence of the time course of fluorescence enhancement and photobleaching during irradiation of ethidium bromide-stained nuclei," Eur. J. Histochem. 44(4), 419-432 (2000).

[19] Naydenova, I., Jallapuram, R., Howard, R., Martin, S. and Toal, V., "Investigation of the diffusion processes in a self-processing acrylamide-based photopolymer system," Appl. Optics 43(14), 2900-2905 (2004). 